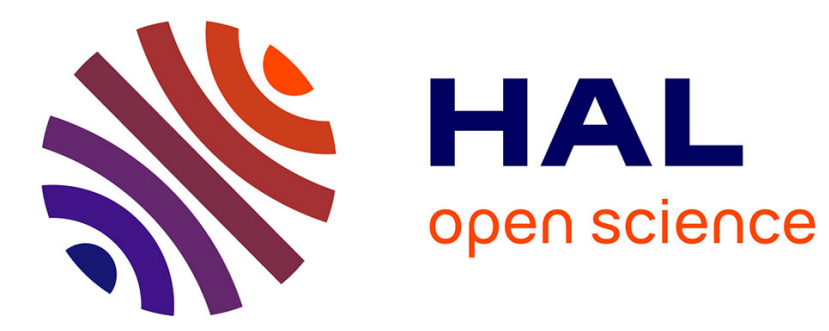

\title{
Étude théorique de divers processus atomiques de croissance sur les surfaces métalliques
}

M. Desjonquères, D. Spanjaard

\section{To cite this version:}

M. Desjonquères, D. Spanjaard. Étude théorique de divers processus atomiques de croissance sur les surfaces métalliques. Journal de Physique IV Proceedings, 1996, 06 (C7), pp.C7-19-C7-30. 10.1051/jp4:1996703 . jpa-00254491

\section{HAL Id: jpa-00254491 https://hal.science/jpa-00254491}

Submitted on 1 Jan 1996

HAL is a multi-disciplinary open access archive for the deposit and dissemination of scientific research documents, whether they are published or not. The documents may come from teaching and research institutions in France or abroad, or from public or private research centers.
L'archive ouverte pluridisciplinaire HAL, est destinée au dépôt et à la diffusion de documents scientifiques de niveau recherche, publiés ou non, émanant des établissements d'enseignement et de recherche français ou étrangers, des laboratoires publics ou privés. 


\title{
Étude théorique de divers processus atomiques de croissance sur les surfaces métalliques
}

\author{
M.C. Desjonquères et $D$. Spanjaard* \\ CEA/DSM/DRECAM/SRSIM, CE de Saclay, 91191 Gif-sur-Yvette, France \\ * Laboratoire de Physique des Solides, Bâtiment 510, Université Paris-Sud, Centre d'Orsay, 91405 Orsay, \\ France
}

\begin{abstract}
Résumé : L'intérêt technologique des matériaux multicouches et la nécessité de comprendre, et si possible de maîtriser, les processus atomiques qui se produisent lors de leur élaboration a suscité depuis quelques années un regain d'activité sur les mécanismes élémentaires intervenant dans la croissance cristalline. Ces processus atomiques sont nombreux. Pour former une nouvelle couche à faible sursaturation, les atomes venant de la vapeur doivent d'abord se condenser sur le substrat, puis diffusent sur la surface et soit s'incorporent aux marches, soit se réévaporent. A sursaturation plus élevée, des îlots se forment par nucléation sur les terrasses du cristal et deviennent eux-mêmes des centres pour la croissance. A chacun de ces processus sont associées des énergies caractéristiques qu'il convient de déterminer si l'on veut faire des études statistiques. Etant donné la réduction ou même l'absence de symétrie des systèmes considérés, on a le plus souvent recours à des méthodes approchées pour calculer ces énergies : théorie du milieu effectif, méthode des liaisons fortes, « Embedded Atom Model » (EAM)...Après avoir décrit brièvement ces méthodes, nous les illustrerons sur une série d'exemples : détermination du site d'adsorption et stabilité des petits îlots de deux et trois atomes sur la surface (111) des métaux de transition cubiques à faces centrées, mécanismes de diffusion superficielle, influence de la présence de marches sur la diffusion.
\end{abstract}

\section{INTRODUCTION}

La croissance cristalline a toujours été d'un grand intérêt fondamental et technologique. Elle vient, durant ces dernières années, de connaître une nouvelle impulsion grâce au développement de méthodes comme l'épitaxie par jets moléculaires qui permettent de réaliser le dépôt de couches très fines sur des substrats solides conduisant ainsi à l'élaboration de dispositifs très utiles en microélectronique, enregistrement magnétique...Mieux on comprendra les processus fondamentaux intervenant lors de la croissance, meilleures seront la qualité et les performances de ces dispositifs.

Certains de ces processus atomiques avaient été déjà envisagés par Stranski et Kossel dans les années vingt et plus tard par Volmer [1]. Toutefois les premières théories atomiques de la croissance remontent aux années cinquante avec, en particulier, la parution de l'article de Burton, Cabrera et Frank [2]. Mais ce n'est que depuis ces vingt dernières années que des techniques nouvelles telles que la microscopie à effet tunnel ou le développement de techniques plus anciennes comme la microscopie ionique de champ ont permis d'observer les éléments fondamentaux de la morphologie d'une surface comme les terrasses, les marches, les crans, les adatomes... La confrontation de ces observations avec les théories précédentes a permis de confirmer ou d'infirmer les mécanismes de croissance supposés et, éventuellement, d'en proposer d'autres.

La première étape à comprendre est l'attachement d'un adatome (condensation) sur une surface plate. On s'est longtemps demandé si l'adatome était instantanément adsorbé dans le site d'adsorption le plus proche de son point d'impact sur la surface ou si son énergie cinétique était dissipée en partie par une migration sur la surface (mobilité transitoire). Cette dernière possibilité a été maintenant écartée à la lumière de diverses simulations en dynamique moléculaire [3]. Pour cette étape il faut donc connaître les divers sites d'adsorption possibles et les énergies d'adsorption correspondantes. Sous l'effet de la température les adatomes peuvent diffuser ce qui leur permet, d'une part, de trouver le site d'adsorption le plus stable et en particulier de s'attacher aux marches et, d'autre part, de se lier à d'autres adatomes. 
Cette phase (nucléation) conduit à la formation de dimères, trimères...Puis les îlots formés peuvent croître soit par incorporation d'atomes diffusant sur la surface, soit par l'arrivée d'atomes incidents sur ces îlots. C'est la phase de croissance. On classe généralement les processus de croissance en trois catégories (Fig. 1):

i)la croissance de type Frank-Van der Merwe qui procède par monocouches successives.

ii)la croissance de type Volmer-Weber où les îlots croissent de façon tridimensionnelle.

iii)la croissance de type Stranski-Krastanov où la croissance initiale est de type Frank-Van der Merwe et se poursuit par une croissance de type Volmer-Weber.

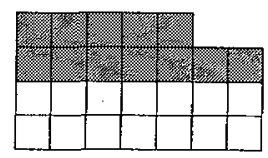

(a)

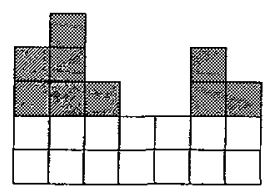

(b)

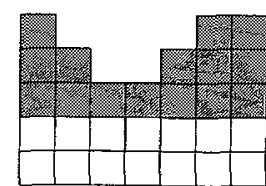

(c)

Figure 1 : Les modes de croissance : a) Frank-Van der Merwe, b) Volmer-Weber, c) Stranski-Krastanov

Le mode de croissance recherché dans la plupart des applications est le type Frank-Van der Merwe qui ne pourra être réalisé que si un atome s'approchant du bord d'une marche ou du bord d'un îlot est capable de descendre dans le plan atomique inférieur.

L'étude de la morphologie de la surface et de son évolution en fonction de la température nécessite des méthodes statistiques dont les paramètres d'entrée sont les énergies mises en jeu dans les divers processus : énergies d'adsorption, de diffusion, d'interaction adatome-marche...Pour calculer ces énergies on a le plus souvent recours à des méthodes semi-empiriques, les méthodes ab-initio se révélant très lourdes à cause de l'absence de symétrie et n'ayant pour l'instant été utilisées que pour les métaux simples [4]. Après avoir brièvement rappelé les principes de ces méthodes, nous les illustrerons par quelques exemples.

\section{METHODES}

Diverses méthodes simplifiées ont été développées pour calculer l'énergie totale d'un système métallique en l'absence de symétrie. Elles peuvent se diviser en deux grandes familles, d'une part les méthodes semiempiriques dans lesquelles l'aspect quantique est pris en compte, d'autre part les méthodes empiriques qui utilisent une expression analytique explicite de l'énergie dont la forme s'inspire des méthodes précédentes. Il est impossible de passer en revue l'ensemble de ces méthodes et nous nous limiterons à présenter brièvement $i$ ) la méthode du milieu effectif basée sur le modèle du jellium traité par la théorie de la fonctionnelle de la densité locale (TFDL) et qui est donc particulièrement adaptée aux métaux dont les électrons de valence sont très délocalisés, $i$ ) la méthode des liaisons fortes qui est appropriée au cas contraire. Nous citerons ensuite brièvement les méthodes empiriques.

\subsection{La théorie du milieu effectif}

L'idée centrale de cette théorie [5] est très simple : il s'agit de remplacer le milieu réel de basse symétrie par un milieu effectif de symétrie élevée, donc beaucoup plus facile à traiter, mais aussi voisin que possible du milieu réel. L'énergie totale d'un atome du métal considéré est alors égale à l'énergie d'immersion de cet atome dans le milieu effectif corrigée par des termes qui rendent compte de la différence entre le milieu réel et le milieu effectif. Etant donné le choix fait pour le milieu effectif, ces termes correctifs sont faibles et peuvent être traités en perturbation. 
Dans la théorie la plus simple on choisit pour milieu effectif relatif à chaque atome $\mathrm{i}$ un jellium homogène dont la densité est une moyenne de la densité électronique provenant des atomes voisins, cette moyenne étant effectuée sur une sphère neutre $S_{i}$ de rayon $S_{\mathrm{i}}$ centrée sur l'atome i considéré. Dans un système inhomogène, à chaque atome $\mathrm{i}$ correspond donc une densité de jellium $\overline{\mathrm{n}}_{i}$ et un rayon $\mathrm{s}_{\mathrm{i}}$ déterminés par l'environnement de cet atome. Moyennant certaines prescriptions pour calculer les densités $\overline{\mathrm{n}}_{i}$ on peut montrer, à partir de la TFDL, que l'énergie totale du système est donnée par l'expression suivante :

$$
\begin{aligned}
\mathrm{E}_{\mathrm{tot}} & =\sum_{i}\left[\mathrm{E}_{\mathrm{hom}}\left(\overline{\mathrm{n}}_{i}\right)-\alpha\left(\overline{\mathrm{n}}_{i}\right) \overline{\mathrm{n}}_{i}+\Delta \mathrm{E}_{\mathrm{AS}}^{\mathrm{i}}\right]+\Delta \mathrm{E}_{\mathrm{lel}} \\
& =\sum_{i} \mathrm{E}_{\mathrm{i}}+\Delta \mathrm{E}_{\text {lel }}
\end{aligned}
$$

Dans cette expression $\mathrm{E}_{\mathrm{hom}}\left(\overline{\mathrm{n}}_{i}\right)$ est l'énergie d'immersion de l'atome i dans le jellium de densité $\overline{\mathrm{n}}_{i}$. Le deuxième terme est une première correction due à la différence entre milieu réel et milieu effectif. Elle provient de l'absence dans le milieu réel de charges positives à l'intérieur de la sphère $S_{\mathrm{i}}$ où on place l'atome i mais qui sont présentes dans le jellium effectif. La fonction $E_{c}(\bar{n})=E_{\text {hom }}(\bar{n})-\alpha(\bar{n}) \bar{n}$ peut être calculée une fois pour toutes. La quantité $\Delta \mathrm{E}_{\mathrm{AS}}^{\mathrm{i}}$ résulte des interactions électrostatiques dues aux recouvrements des sphères neutres. Dans un solide compact ce recouvrement est considéré comme négligeable mais lorsque la coordinence diminue les rayons $\mathrm{s}_{\mathrm{i}}$ augmentent, et il doit être pris en compte. Jacobsen et al. [5] ont montré que ce terme peut s'écrire sous la forme suivante, moyennant certaines approximations assez bien justifiées :

$$
\Delta \mathrm{E}_{\mathrm{AS}}^{\mathrm{i}}=\alpha\left(\overline{\mathrm{n}}_{i}\right) \overline{\mathrm{n}}_{i}+(1 / 2) \sum_{j \neq i} \mathrm{~V}\left(\left|\mathbf{R}_{\mathrm{i}}-\mathbf{R}_{\mathrm{j}}\right|\right)
$$

où $V$ est un potentiel de paire dont l'expression analytique s'obtient en écrivant que $\Delta \mathrm{E}_{\mathrm{AS}}^{\mathrm{i}}$ est nul dans le volume d'un solide compact. Enfin le dernier terme $\Delta \mathrm{E}_{1 \mathrm{el}}$ est dû à la différence, entre milieu réel et milieu effectif, de la somme des énergies à un électron occupées. Ce terme joue un rôle important dans les métaux de transition mais il est faible dans les métaux à bande de valence $s p$ sauf si le système possède des atomes à faible coordinence comme nous le verrons dans l'un des exemples que nous présenterons.

La fonction $\mathrm{E}_{\mathrm{c}}(\overline{\mathrm{n}})$ qui passe par un minimum pour $\overline{\mathrm{n}}=\mathrm{n}_{0}$ décrit donc la variation de l'énergie d'un métal $s p$ compact en fonction de la densité $\overline{\mathrm{n}}$ du jellium effectif (qui est la même pour tous les atomes) c'est à dire du volume atomique et détermine donc le paramètre de réseau, l'énergie de cohésion par atome et le module de compressibilité. Lorsque le système est inhomogène et que $\Delta \mathrm{E}_{\text {1el }}$ est négligeable, on peut montrer que la contribution à l'énergie totale de chaque atome i s'exprime analytiquement en fonction des distances interatomiques avec ses voisins et d'un certain nombre de paramètres qui sont soit calculés à priori, soit ajustés à des grandeurs expérimentales. Une telle approche est donc très bien adaptée à des calculs de simulations.

\subsection{La méthode des liaisons fortes}

Dans cette méthode [6] on décompose l'énergie totale en deux contributions :

$$
E_{\text {tot }}=E_{\text {bande }}+E_{\text {rep }}
$$

où $E_{\text {bande }}$ provient de l'élargissement des niveaux de valence du métal en une bande d'énergie partiellement remplie. Dans les métaux de transition ce terme est essentiellement dû à la formation de la bande $d$, les électrons $s p$ ne jouant un rôle non négligeable dans l'énergie de cohésion que pour les éléments dont la bande $d$ est presque vide ou pleine. Nous ne considérerons donc que les électrons $d$ qui, étant relativement localisés, sont bien décrits par une méthode de liaisons fortes. L'élargissement des niveaux abaisse 
l'énergie du système et ceci d'autant plus que cet élargissement est grand, c'est à dire que les atomes sont proches. $E_{b a n d e}$ décrit ainsi la partie attractive de l'énergie. Il est donc nécessaire d'ajouter un terme répulsif $E_{\text {rep }}$ qui assure l'équilibre du système. Ce terme qui a tout d'abord été introduit de façon phénoménologique sous la forme d'un potentiel de paire, a reçu une justification dérivée de la TFDL [7]. $\mathrm{E}_{\text {rep }}$ est dû essentiellement aux interactions électron-électron, qui s'opposent à l'interpénétration des nuages électroniques, et aux répulsions cœur-cœur. On l'écrit, le plus souvent, comme une somme d'interactions de type Born-Mayer [8] :

$$
\mathrm{E}_{\mathrm{rep}}=\sum_{i, j<i} \mathrm{~A} \exp \left[-\mathrm{p}\left(\mathrm{R}_{\mathrm{ij}} / \mathrm{R}_{0}-1\right)\right]
$$

où $R_{i j}$ est la distance entre les atomes $i$ et $j, R_{0}$ est une distance de référence généralement égale à la distance entre premiers voisins à l'équilibre, la somme sur $i$ et $j$ est limitée aux premiers ou aux seconds voisins selon que la structure est compacte ou cubique centrée. A et $\mathrm{p}$ sont des paramètres caractéristiques du métal.

Le calcul du terme $\mathrm{E}_{\text {bande }}$ nécessite la détermination de la densité d'états électroniques du système. Dans la méthode des liaisons fortes, on décompose les états électroniques $|n\rangle$, d'énergie $E_{n}$, sur une base d'orbitales atomiques $\mid \mathrm{i} \lambda>\left(\lambda=\mathrm{xy}, \mathrm{yz}, \mathrm{zx}, \mathrm{x}^{2}-\mathrm{y}^{2}, 3 \mathrm{z}^{2}-\mathrm{r}^{2}\right.$ pour les orbitales $\left.d\right)$ centrées sur chaque site i :

$$
\mathrm{n}>=\sum_{i, \lambda} \mathrm{c}_{\mathrm{i} \lambda}\left(\mathrm{E}_{\mathrm{n}}\right)|\mathrm{i} \lambda\rangle
$$

La base $\mid \mathrm{i} \lambda>$ est supposée orthonormale et complète. Dans cette base, l'hamiltonien $\mathrm{H}$ est déterminé par : i)des éléments de matrice intraatomiques $\langle\mathrm{i} \lambda|\mathrm{H}| i \lambda\rangle=\mathrm{E}_{\mathrm{i}}$, c'est à dire les niveaux atomiques effectifs qui, dans le volume, sont tous égaux et définissent le centre de gravité de la bande $d$ qu'il est commode de choisir pour référence d'énergie.

ii)des éléments de matrice interatomiques $\langle\mathrm{i} \lambda|\mathrm{H}| \mathrm{j} \mu\rangle$ appelés intégrales de saut qui dépendent de la direction de liaison $\mathbf{R}_{\mathrm{ij}} / \mathbf{R}_{\mathrm{ij}}$ et des trois paramètres de Slater-Koster [9] dd $\lambda(\lambda=\sigma, \pi, \delta)$ dont la variation avec la distance $R_{i j}$ est supposée exponentielle :

$$
\mathrm{dd} \lambda=\mathrm{dd} \lambda_{0} \exp \left[-\mathrm{q}\left(\mathrm{R}_{\mathrm{ij}} / \mathrm{R}_{0}-1\right)\right]
$$

Ces interactions sont limitées aux premiers ou aux seconds voisins. Les coefficients $\mathrm{dd} \lambda_{0}$ sont déduits de calculs de structure de bande ab-initio et $q \cong 3-5$.

Pour le cristal volumique, les états propres et la densité d'états par atome $\mathrm{n}_{0}(\mathrm{E})$ peuvent être obtenus facilement en utilisant le théorème de Bloch. On peut aussi calculer la densité d'états directement par la méthode des fractions continues qui ne fait pas appel à la périodicité du réseau $[6,10]$. L'énergie de bande totale est donnée par :

$$
\mathrm{E}_{\mathrm{bande}}=\mathrm{N} \int^{E_{F}} E n_{0}(E) d E
$$

où $E_{F}$ est le niveau de Fermi et $\mathrm{N}$ le nombre d'atomes. En ajoutant le terme répulsif on obtient la variation de l'énergie totale en fonction de la distance interatomique. Il est alors possible de déterminer les paramètres $\mathrm{A}$ et $\mathrm{p}$ de manière à reproduire la valeur expérimentale du volume atomique et du module de compressibilité, par exemple.

Pour décrire la modification de la structure électronique due à un défaut, il est commode d'introduire le concept de densité d'états locale au site i : 


$$
\begin{aligned}
\mathrm{n}_{\mathrm{i}}(\mathrm{E}) & =2 \sum_{i, \lambda}\left|\mathrm{c}_{\mathrm{i \lambda}}\left(\mathrm{E}_{\mathrm{n}}\right)\right|^{2} \delta\left(E-E_{\mathrm{n}}\right) \\
& =\mathrm{n}_{0}(\mathrm{E})+\Delta \mathrm{n}_{\mathrm{i}}(\mathrm{E})
\end{aligned}
$$

où $\Delta \mathrm{n}_{\mathrm{i}}$ (E) est la perturbation de la densité d'états locale au site i qui n'est appréciable que dans le voisinage immédiat du défaut et peut être calculée avec la méthode des fractions continues. Cette perturbation induit une redistribution de charge qui modifie le potentiel sur les atomes proches du défaut, et donc le niveau atomique effectif, d'une quantité $\Delta V_{i}$. Les quantités $\Delta V_{i}$ et la redistribution des charges correspondante devraient être calculées de façon autocohérente. Cependant, dans un métal, les charges sont écrantées sur une distance de l'ordre de la distance interatomique et donc les quantités $\Delta \mathrm{V}_{\mathrm{i}}$ sont déterminées par une condition de neutralité de charge locale :

$$
\int^{E_{\mathrm{r}}} \mathrm{n}_{\mathrm{i}}\left(\mathrm{E}, \ldots, \Delta \mathrm{V}_{\mathrm{j}, \ldots)}, \ldots \mathrm{E}=\mathrm{N}_{\mathrm{d}}\right.
$$

où $\mathrm{N}_{\mathrm{d}}$ est le nombre d'électrons par atome dans la bande $d$ du métal. L'énergie du défaut s'écrit donc :

$$
\begin{aligned}
\Delta \mathrm{E}_{\mathrm{tot}} & =\Delta \mathrm{E}_{\text {bande }}+\Delta \mathrm{E}_{\text {rep }} \\
& =\sum_{i \in C}\left[\int^{\mathrm{E}_{\mathrm{F}}} \mathrm{E} \Delta \mathrm{n}_{\mathrm{i}}(\mathrm{E}) \mathrm{dE}-\mathrm{N}_{\mathrm{d}} \Delta \mathrm{V}_{\mathrm{i}}\right]+\Delta \mathrm{E}_{\text {rep }}
\end{aligned}
$$

$\mathrm{C}$ est l'ensemble des atomes où la perturbation due au défaut est appréciable et $\Delta \mathrm{E}_{\mathrm{rep}}$ est la modification de la somme des interactions répulsives de paires. Le deuxième terme dans le crochet évite de compter deux fois la variation des interactions électron-électron qui sont responsables du déplacement du niveau atomique $\Delta \mathrm{V}_{\mathrm{i}}$.

La précision avec laquelle doit être calculée $\Delta \mathrm{n}_{\mathrm{i}}(\mathrm{E})$ dépend de l'ordre de grandeur de $\Delta \mathrm{E}_{\text {tot }}$; plus la précision demandée est grande, plus le nombre de moments exacts $\mu_{\mathrm{p}}^{\mathrm{i}}$ :

$$
\mu_{p}^{i}=\int_{\infty}^{+\infty} E^{p} n_{i}(E) d E
$$

de la densité d'états $n_{i}(E)$ doit être élevé. On peut montrer que si la fraction continue a n étages exacts, les $2 n$ premiers moments de $n_{i}(E)$ sont aussi exacts.

\subsection{Les méthodes empiriques}

Il existe dans la littérature de nombreuses expressions empiriques pour modéliser l'énergie totale des systèmes métalliques en fonction des coordonnées des atomes constituants. On s'est vite aperçu qu'un modèle d'interactions de paires ne rendait pas bien compte des propriétés énergétiques non seulement des métaux, mais aussi des covalents. C'est pourquoi depuis ces dix dernières années ont été proposés de nouveaux modèles s'affranchissant de cette approximation en s'inspirant de la théorie du milieu effectif ou de celle des liaisons fortes. Dans toutes ces méthodes l'énergie totale est écrite sous la forme :

$$
\mathrm{E}_{\mathrm{tot}}=\sum_{i} \quad \mathrm{E}_{\mathrm{i}}=\sum_{i}\left[\mathrm{~F}\left(\sum_{j \neq i} \mathrm{f}\left(\mathrm{R}_{\mathrm{ij}}\right)\right)+(1 / 2) \sum_{j \neq i} \mathrm{~V}\left(\mathrm{R}_{\mathrm{ij}}\right)\right]
$$


où $V\left(R_{i j}\right)$ est un potentiel de paire. Les modèles diffèrent entre eux par les formes fonctionnelles de $F$ et $f$ et par leur interprétation. Dans les modèles dérivés de la théorie du milieu effectif ( Embedded Atom Model (EAM) [11], glue model [12]...) l'argument de la fonction F est interprété comme une densité électronique locale provenant des atomes voisins de l'atome $i$. Les modèles inspirés de la méthode des liaisons fortes partent d'une approximation où la densité d'états locale est caractérisée par son seul second moment centré $\mu_{2}^{\text {ic }}$. La contribution de l'atome $\mathrm{i}$ au terme de bande est proportionnelle à la largeur moyenne de la densité d'états locale $\mathrm{n}_{\mathrm{i}}(\mathrm{E})$, c'est à dire à $\left(\mu_{2}^{\mathrm{ic}}\right)^{1 / 2}$. Ceci suggère de choisir pour fonction $\mathrm{F}$ une racine carrée dont l'argument est proportionnel à $\mu^{\text {ic }}{ }_{2}$. De plus on peut montrer facilement que :

$$
\left.\mu_{2}^{\mathrm{ic}}=2 \sum_{j \neq i, \lambda, \mu}<\mathrm{i} \lambda|H| j \mu><\mathrm{j} \mu|H| \mathrm{i} \lambda\right\rangle
$$

L'argument de la fonction F s'interprète donc comme une somme de carrés d'intégrales de saut du site i aux sites voisins $\mathrm{j}$, à une constante multiplicative près. Ce point de vue a été adopté par Finnis et Sinclair [13] et Rosato et al. [14], les potentiels proposés ne différant que par le choix des fonctions $f$ et $V$.

Tous ces potentiels contiennent des paramètres ajustables qui sont déterminés empiriquement de façon à reproduire des quantités physiques connues: énergie de cohésion, volume atomique, constantes élastiques...

\section{ETUDE ENERGETIQUE DE QUELQUES PROBLEMES INTERVENANT LORS DE LA CROISSANCE CRISTALLINE}

\subsection{Croissance des îlots sur la surface (111) des métaux de transition CFC}

L'homoépitaxie sur la face (111) des métaux de transition CFC pose des problèmes intéressants. D'une part, il existe sur cette face deux sites d'adsorption possibles : le site normal $\mathrm{N}$ ou CFC qui continue l'empilement régulier $\mathrm{CFC}$, et le site en faute $\mathrm{F}$ ou $\mathrm{HC}$ autour duquel la croissance induirait une faute d'empilement. D'autre part, lorsque leur taille croît les îlots peuvent développer deux sortes de bords compacts correspondants à deux types de microfacettes : (001) pour le type A et (111) pour le type B.

Le problème de la stabilité relative des sites $\mathrm{N}$ et $\mathrm{F}$ est clairement relié à celui de la stabilité relative des phases CFC et $\mathrm{HC}$ en volume dont on sait qu'elle est déterminée par le remplissage de la bande $d$ [15]. Dans des études précédentes [16] nous avions montré que pour certains remplissages $\mathrm{N}_{\mathrm{d}}$ un adatome occupe un site $\mathrm{F}$ alors que la couche complète n'introduit pas de faute d'empilement. On prévoit donc une inversion de stabilité entre les deux sites quand la taille de l'îlot croît. Nous avons étudié [17] la stabilité relative des monomères, dimères et trimères triangulaires adsorbés sur la surface (111) en fonction de $\mathrm{N}_{\mathrm{d}}$ dans le domaine de stabilité des métaux $C F C\left(\mathrm{~N}_{\mathrm{d}}>7.5 \mathrm{e} /\right.$ atome). Toutes les configurations atomiques possibles de ces îlots ont été considérées : monomères et dimères aux sites $\mathrm{N}$ et $\mathrm{F}$, triangles de types $\mathrm{A}$ et $\mathrm{B}$ aux sites $\mathrm{N}$ et $\mathrm{F}$ (Fig 2).

L'énergie d'adsorption de ces îlots est minimisée, d'une part, par rapport à la relaxation verticale des adatomes et, d'autre part, par rapport à un déplacement horizontal de leurs premiers voisins respectant la symétrie du système. Comme on pouvait s'y attendre les adatomes relaxent vers la surface et repoussent leurs premiers voisins. De plus, les amplitudes de ces relaxations sont assez bien différenciées entre site d'adsorption $\mathrm{N}$ et $\mathrm{F}$, mais cette différence diminue lorsque la taille de l'îlot augmente. Par contre, les relaxations sont du même ordre de grandeur pour les triangles $\mathrm{A}$ et $\mathrm{B}$ adsorbés au même site.

Nous avons résumé sur la figure 3 les résultats concernant la stabilité relative de ces îlots. On voit qu'il existe un domaine de $\mathrm{N}_{d}$ dans lequel le site $\mathrm{F}$ est plus stable que le site $\mathrm{N}$ pour le monomère, mais que ce domaine se rétrécit très vite lorsque la taille de l'îlot augmente. Pour ces valeurs de $\mathrm{N}_{\mathrm{d}}$ on prévoit donc une transition du site $\mathrm{F}$ vers le site $\mathrm{N}$ pour des îlots de deux ou trois atomes. On trouve de plus que les triangles $B$ sont plus stables que les triangles $A$ si $N_{d} \leq 8$ e/atome et ceci quel que soit le site d'adsorption. Ces résultats peuvent être comparés avec les expériences de microscopie ionique sur Ir réalisées par Wang 
et Ehrlich [18]. En prenant $\mathrm{N}_{\mathrm{d}}=7.5-7.6 \mathrm{e}$ /atome pour cet élément, nous prévoyons, en excellent accord avec les expériences, que les monomères et les dimères occupent des sites $F$ alors que les trimères occupent indifféremment les sites $\mathrm{F}$ et $\mathrm{N}$, mais les triangles $\mathrm{B}$ sont notablement plus stables que les triangles $\mathrm{A}$. Notons que les effets de relaxation, étant assez différents aux sites $\mathrm{N}$ et $\mathrm{F}$, jouent un rôle important dans leur stabilité relative.
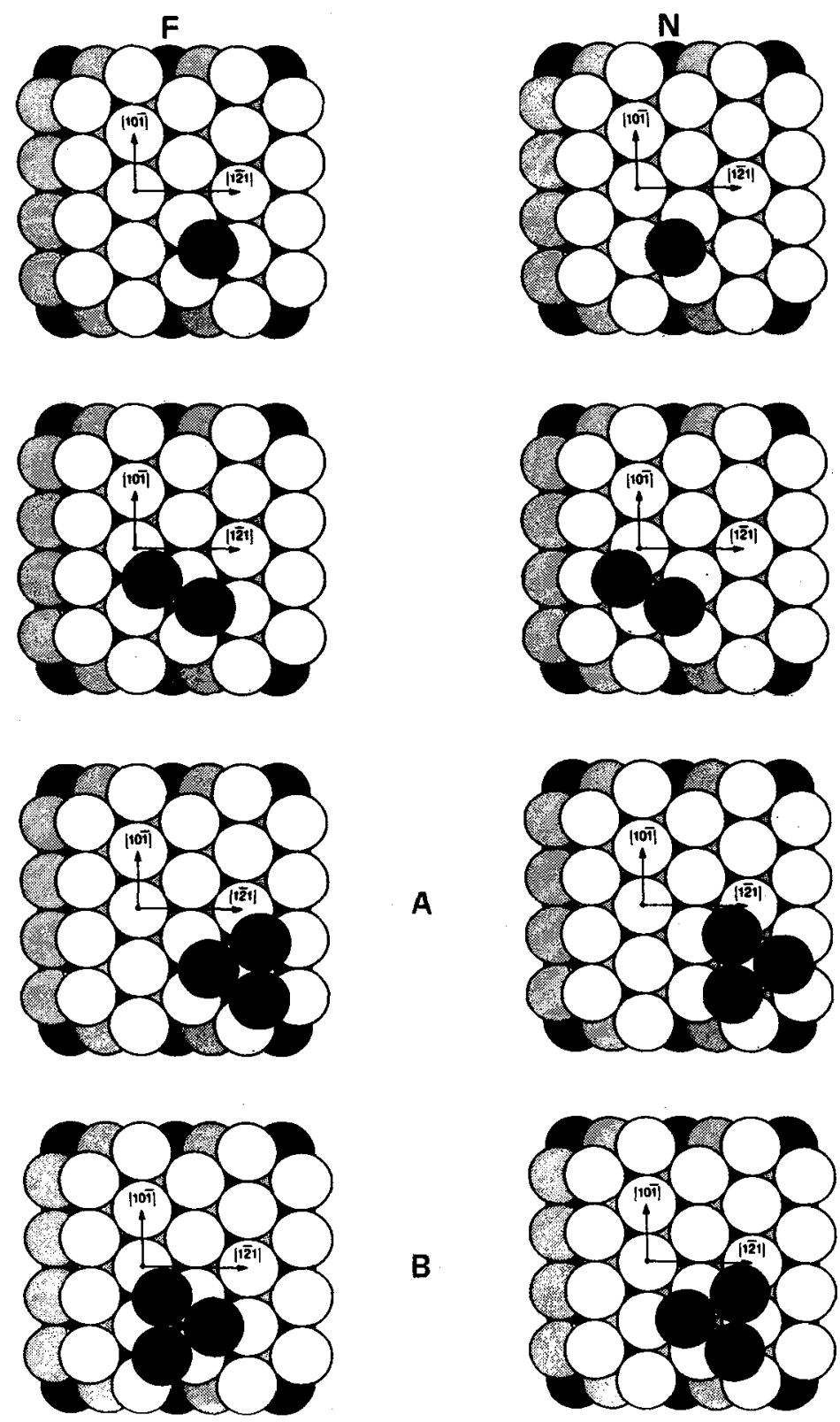

A

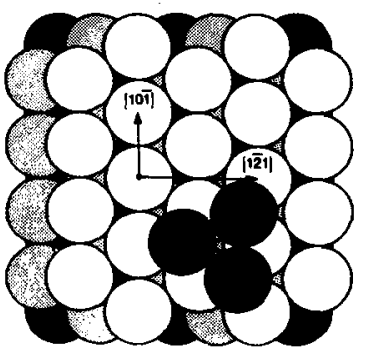

Figure 2 : Les configurations atomiques des monomères, dimères et trimères triangulaires sur la face (111) d'un cristal CFC aux sites normaux $(N)$ et en faute $(F)$. Les trimères de type $A$ ont leur centre au dessus d'un atome de surface et présentent des microfacettes de type (001). Les trimères de type $B$ ont leur centre sur un site d'adsorption et présentent des microfacettes de type (111). 


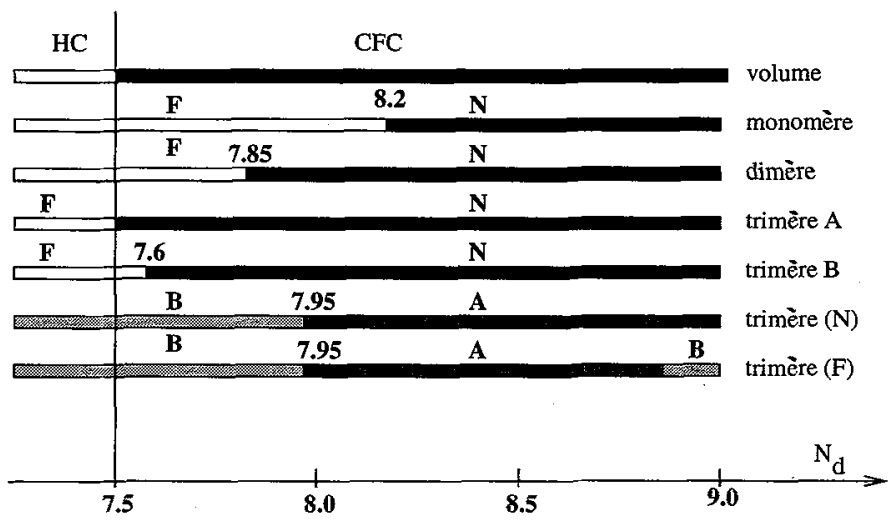

Figure 3 : Diagramme donnant la stabilité relative des différentes configurations atomiques montrées sur la Figure 2 en fonction du remplissage de la bande $d\left(\mathrm{~N}_{d}\right)$. De la deuxième à la cinquième ligne : stabilité relative des sites $\mathrm{N}$ et $\mathrm{F}$ pour les monomères,dimères, trimères $\mathrm{A}$ et trimères $\mathrm{B}$. Sixième et septième ligne : stabilité relative des triangles $\mathrm{A}$ et $\mathrm{B}$ aux sites $\mathrm{N}$ et F. La stabilité relative des phases volumiques $\mathrm{HC}$ et CFC est donnée dans la première ligne pour comparaison.

\subsection{Diffusion superficielle}

La diffusion des adatomes sur les surfaces est non seulement le paramètre essentiel contrôlant la croissance mais aussi elle reflète la nature des barrières et des potentiels d'interaction sur les surfaces. Il a été longtemps supposé que les adatomes se déplaçaient par une série de sauts entre sites d'adsorption adjacents. Contrairement à cette image intuitive on sait maintenant que pour certaines surfaces le mécanisme de diffusion superficielle peut être beaucoup plus compliqué. De plus la présence de défauts, tels que des marches, sur la surface introduit une anisotropie dans la diffusion et éventuellement des barrières supplémentaires. Nous allons illustrer chacun de ces points par un exemple.

\subsubsection{Mécanisme de diffusion sur les surfaces plates}

Considérons d'abord les surfaces les plus compactes (110) CC et (111) CFC. Des calculs d'énergie d'activation pour une diffusion superficielle par saut ont été effectués aussi bien par la méthode des liaisons fortes que par celle du milieu effectif. Sur la figure 4 nous montrons les résultats d'un calcul de liaisons fortes [19] pour la diffusion d'adatomes de la série $5 d$ sur la face (110) du tungstène comparés avec des résultats expérimentaux obtenus en microscopie ionique [20]. L'accord théorie-expérience est tout à fait raisonnable. Il existe également des calculs de diffusion par saut sur la surface (111) CFC effectués à l'aide de la théorie du milieu effectif [21] ou en EAM [22].

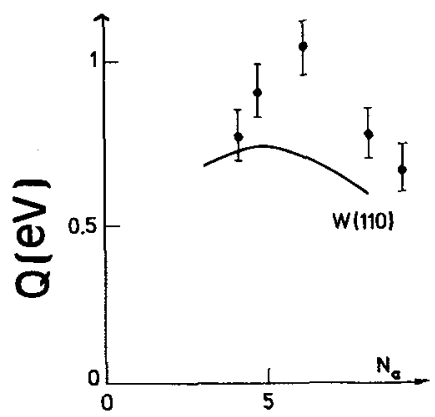

Figure 4 : Energie d'activation pour la diffusion (extrapolée a $0 \mathrm{~K}$ ) d'adatomes $5 d(\mathrm{Ta}, \mathrm{W}, \mathrm{Re}, \mathrm{Ir}, \mathrm{Pt}$ ) sur la face (110) du tungstène comparée aux expériences de la référence [20] 
Il faut remarquer que les énergies d'activation pour la diffusion par saut sur les surfaces les plus compactes sont relativement modérées $\left(\approx\right.$ quelques $10^{-1} \mathrm{eV}$ ) mais deviennent beaucoup plus importantes sur les surfaces plus ouvertes car le nombre de liaisons coupées lors de la diffusion est plus grand. On peut donc penser que des mécanismes de diffusion moins coûteux en énergie pourraient se produire sur ces surfaces. Un tel mécanisme, appelé mécanisme d'échange, a été observé sur la face (100) de certains éléments CFC ( Ir, Pt, Re sur Ir,...) en microscopie ionique [23]. En effet dans ces systèmes on observe que l'adatome effectue une marche au hasard sur un réseau carré $c(2 \times 2)$, c'est à dire que sur le réseau carré de la surface l'atome ne peut se déplacer qu'en diagonale (Fig. 5). De plus cette méthode permet de déterminer la nature de l'adatome qui diffuse et on observe que si l'on dépose un atome A sur un substrat B l'adatome a changé de nature après diffusion. Ces faits expérimentaux peuvent s'expliquer par le mécanisme de diffusion suivant (Fig. 6a) : l'adatome pousse un adatome du substrat sur la surface pour prendre sa place dans le réseau du premier plan. De cette manière l'adatome garde plus de liaisons avec ses voisins que dans un processus de saut ce qui peut abaisser l'énergie d'activation pour la diffusion. Notons qu'un mécanisme similaire avait déjà été proposé dans les années soixante dix pour expliquer la diffusion intercanal dans les surfaces à canaux comme la surface (011) du Platine [24] mais, jusqu'au début des années quatre vingt dix, une telle possibilité n'avait pas été envisagée pour des surfaces aussi compactes que (100) CFC.

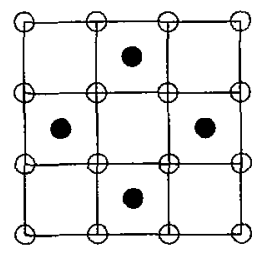

Figure 5 : Carte des sites visités (cercles pleins) lors de la diffusion par échange d'un adatome sur une surface (100) CFC (cercles vides : atomes de surface).

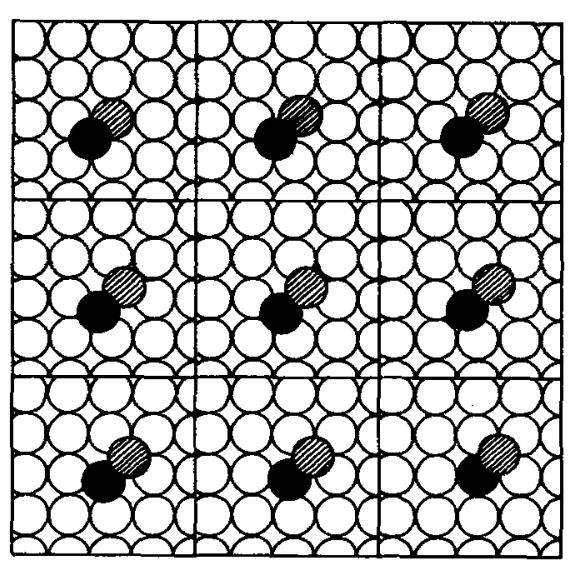

(a)

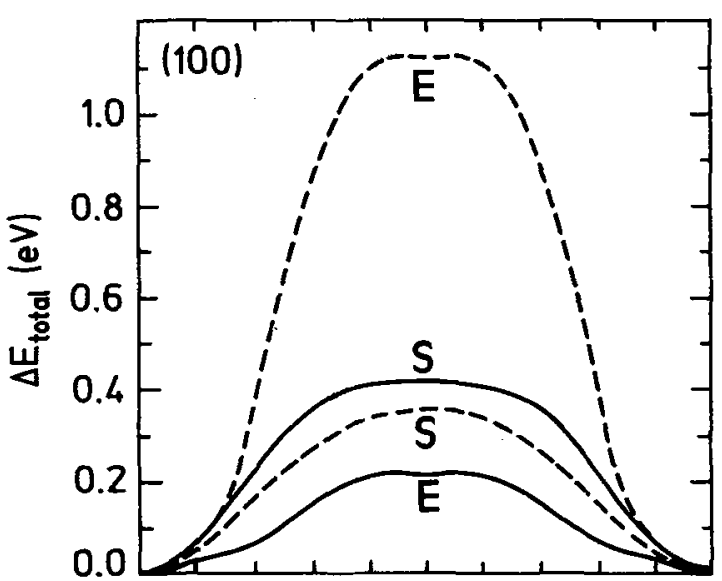

(b)

Figure 6 : a) Déplacements atomiques lors d'une diffusion par échange sur la surface (100) d'un cristal CFC. b) Barrières de potentiel rencontrées par un adatome de Cu lors de sa diffusion sur $\mathrm{Cu}(100)$ d'après Hansen et al. [26] obtenues, d'une part, en négligeant $\Delta \mathrm{E}_{\text {lel }}$ (trait discontinu) et, d'autre part, en en tenant compte (trait plein). $\mathrm{S}(\mathrm{E})$ désigne la diffusion par saut(échange). 
Du point de vue théorique, le premier calcul concluant à un mécanisme de diffusion par échange est dû à Feibelman [25] dans le cas de l'autodiffusion d'aluminium sur $\mathrm{Al}(100)$, étudié dans le cas de la TFDL. Depuis plusieurs calculs ont été effectués utilisant la théorie du milieu effectif. Dans cette théorie, le mécanisme de diffusion par saut reste le plus favorable lorsque l'on néglige la contribution du terme à un électron. Dans la géométrie particulière correspondant au maximum de l'énergie potentielle (Fig. 6a), dans laquelle un dimère est adsorbé sur une lacune, les effets de covalence sont importants. Dans les études effectuées sur $\mathrm{Cu}$ [26] et $\mathrm{Al}$ [27] ces effets de covalence bien que concernant des électrons sp, sont traités en liaisons fortes. Sur $\mathrm{Cu}$ et $\mathrm{Al}(100)$, l'effet du terme à un électron est de relever la hauteur de la barrière pour la diffusion par saut mais de l'abaisser pour la diffusion par échange (Fig. 6b). Enfin ce type de mécanisme explique aussi la diffusion intercanal sur $\mathrm{Al}(011)$ [27].

Notons que lorsque l'on dépose des atomes $\mathrm{A}$ sur un substrat $\mathrm{B}$, la diffusion par échange est l'un des mécanismes intervenant dans la formation d'un alliage de surface.

\subsubsection{Adsorption et diffusion près des marches : effet Schwoebel}

La connaissance du potentiel d'interaction adatome-marche est fondamentale pour comprendre le mécanisme de croissance d'un cristal. Lorsque le taux de recouvrement en adatomes est élevé et que la température est suffisamment haute pour que les atomes puissent diffuser facilement, les îlots formés sont compacts et peuvent avoir un double effet : d'une part, les atomes s'adsorbant sur la surface peuvent, par diffusion, venir s'incorporer à l'îlot qui agit donc ainsi comme un nouveau centre de croissance bidimensionnelle mais, d'autre part, un atome peut aussi se condenser sur un îlot. Dans ce dernier cas il peut exister ou non sur les bords de l'îlot une barrière de potentiel supplémentaire (effet Schwoebel [28]) confinant l'adatome sur l'îlot, du moins si la température n'est pas trop élevée, et induisant ainsi une croissance à trois dimensions.

Nous avons étudié [29] l'énergie potentielle d'un adatome sur une surface à marches [m(110)x(0 11)] d'un métal de transition cubique centré où les terrasses sont d'orientation (110) et la contre-marche d'orientation (011), l'arête de la marche étant alors constituée d'une file dense d'atomes (Fig. 7a). Dans ce calcul l'énergie de liaison d'un adatome, obtenue par la méthode des liaisons fortes, est minimisée par rapport à la coordonnée $\mathrm{z}$ pour des valeurs données de $\mathrm{x}$ et $\mathrm{y}$, l'axe $\mathrm{Oz}$ étant perpendiculaire aux terrasses. Cette minimisation est effectuée dans un domaine de $(x, y)$ incluant la marche. A partir de ces calculs on trace des cartes de l'énergie potentielle de l'adatome d'où l'on déduit les sites d'adsorption les plus stables et leurs énergies de liaison, les chemins et les énergies de diffusion par saut.
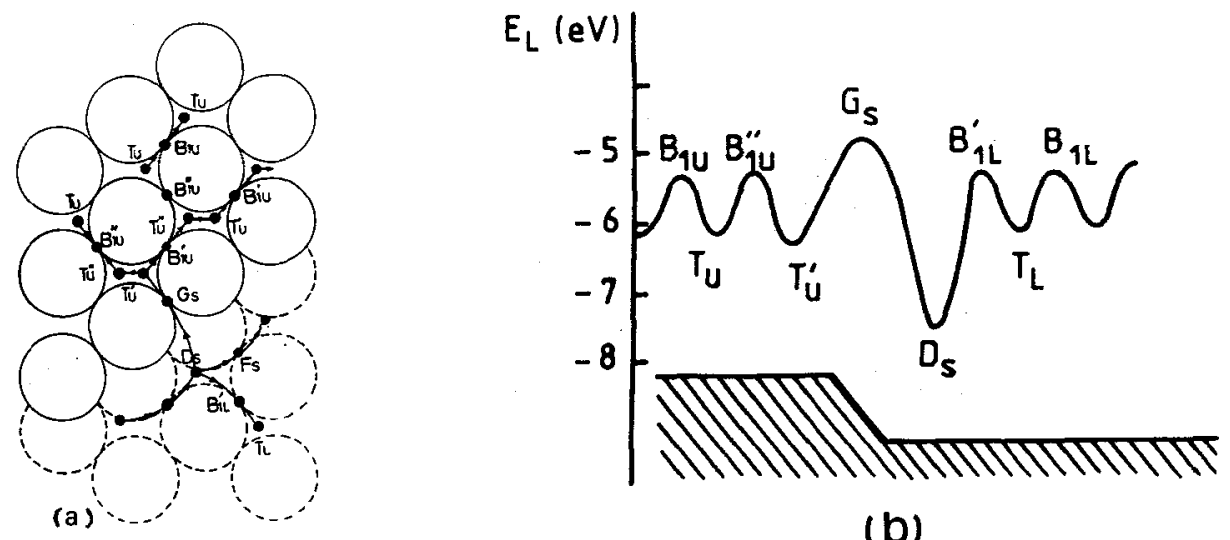

(b)

Figure $7:$ a) Sites et chemins de diffusion par saut sur la surface à marches $[\mathrm{m}(110) \times(011)]$. Les atomes de la terrasse supérieure (inférieure) sont dessinés en trait plein (discontinu). b) Profil d'énergie potentielle d'un adatome de $W$ sur $W$ diffusant à travers la marche. 
L'étude est faite en fonction du remplissage de la bande $d$, dans le domaine de stabilité des cubiques centrés $\left(3<\mathrm{N}_{\mathrm{d}}<7 \mathrm{e}\right.$ //atome). Les tendances sont les mêmes quel que soit $\mathrm{N}_{\mathrm{d}}$. Un exemple typique est donné sur la figure $7 \mathrm{~b}$. On trouve que dans la géométrie d'énergie minimum l'atome est incorporé au bord de la marche où sa coordinence est maximum. Le site suivant en stabilité est sur la terrasse supérieure au bord de la marche. En effet, à cette position, l'adatome possède des liaisons avec les atomes de l'arête qui sont moins coordonnés et contribuent davantage à l'énergie de liaison, la variation de la racine carrée de leur second moment centré étant plus grande (voir 2.2). Enfin, la barrière de potentiel que doit franchir un adatome pour descendre la marche est supérieure de quelques $10^{-1} \mathrm{eV}$ à celle rencontrée lors de la diffusion sur les terrasses car l'adatome doit passer par une position où il a moins de proches voisins que sur un site $B_{1 U}$ (Fig. 7a). Il existe donc un effet Schwoebel sur cette surface. Ceci est en bon accord avec les expériences de Bassett [20] et de Wang et Tsong [30] concernant les adatomes 5d'sur W(110). Par contre l'énergie d'activation pour la diffusion le long de la marche est peu perturbée par celle-ci.

Lorsque l'arête n'est plus une file dense d'atomes et, en particulier, lorsqu'elle présente des crans comme dans le cas de la surface $[\mathrm{m}(110) \times(001)]$ où l'arête est parallèle à la direction $<\overline{1} 10>$, l'effet Schwoebel est toujours présent mais la diffusion le long de la marche devient plus difficile [31].

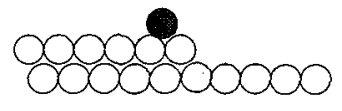

(a)

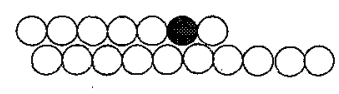

(b)

Figure 8 : Mécanisme de diffusion par échange permettant à un atome de s'incorporer dans une marche.

Notons enfin qu'on peut également imaginer un mécanisme de diffusion par échange permettant à un adatome de descendre la marche (Fig. 8). Ceci a été observé en microscopie ionique [32] dans le cas d'atomes de $\mathrm{W}$ diffusant sur des îlots d'Ir déposés sur la face (111) de ce métal. Cette expérience montre que les rôles respectifs des mécanismes de diffusion par saut ou par échange dépendent de la taille de l'îlot et de la structure de ses bords. Des calculs effectués à l'aide de la théorie du milieu effectif pour un adatome de $\mathrm{Cu}$ sur des îlots de $\mathrm{Cu}$ présents sur $\mathrm{Cu}(111)$ confirment bien ce résultat [21].

\section{CONCLUSION}

En conclusion, le développement de la microscopie à effet tunnel et les progrès effectués dans les techniques d'acquisition de données en microscopie ionique ont permis une meilleure connaissance des mécanismes contrôlant la croissance cristalline au niveau atomique en révélant en particulier qu'un phénomène tel que la diffusion superficielle pouvait être plus compliqué, mais aussi beaucoup plus intéressant, que le simple mécanisme de saut sur une surface quasi-rigide. Ces expériences ont donné une impulsion aux calculs théoriques cherchant à évaluer les paramètres énergétiques intervenant dans ces phénomènes. Toutefois les informations obtenues restent assez éparses et un effort reste à faire pour mettre en évidence des tendances générales.

\section{Références}

[1] Une revue est donnée dans « Crystal growth : an introduction » P. Hartman Ed., (North-Holland, Amsterdam 1973)

[2] Burton W.K., Cabrera N. et Frank F., Phys. Trans. Roy. Soc., 243 (1951) 299-342

[3] Sanders D.E. et De Pristo A.E., Surf. Sci, 254 (1991) 341-353 
Sanders D.E., Halstead D.M. et De Pristo A.E., J. Vac. Sci. Technol. A, 10 (1992) 1986-1992

[4] Feibelman P.J., Phys. Rev. Lett., 63 (1989) 2488-2491

Stumpf R. et Scheffler M., Phys. Rev. Lett., 72 (1994) 254-257

[5] Jacobsen K.W., Norskov J.K. et Puska M.J., Phys. Rev. B, 35 (1987) 7423-7442

Jacobsen K.W., Comm. Cond. Mat. Phys., 14 (1988) 129-161

[6] Desjonquères M.C. et Spanjaard D., Concepts in surface physics (Springer-Verlag, Berlin, 1996)

[7] Foulkes W.M.C. et Haydock R., Phys. Rev. B, 39 (1989) 12520-12536

Sutton A.P., Finnis M.W., Pettifor D.G. et Ohta Y., J. Phys. C, 21 (1988) 35-66

[8] Ducastelle F., J. Phys. France, 31 (1970) 1055-1062

[9] Slater J.C. et Koster G.F., Phys. Rev., 94 (1954) 1498-1524

[10] Haydock R.,Heine V. et Kelly M.J., J. Phys. C, 5 (1972) 2845-2858

[11] Daw M.S. et Baskes M.I., Phys. Rev. Lett., 50 (1983) 1285-1288 ; Phys. Rev. B, 29 (1984) 6443-6453

[12] Ercolessi F., Tossati E. et Parrinello M., Phys. Rev. Lett., 57 (1986) 719-722

[13] Finnis M.W. et Sinclair J.E., Phil. Mag. A, 50 (1984) 45-55

[14] Rosato V.M., Guillopé M. et Legrand B., Phil. Mag. A, 59 (1989) 321-336

[15] Ducastelle F., Order and stability in alloys, 3 (North-Holland, Amsterdam, 1991)

[16] Piveteau B., Desjonquères M.C. et Spanjaard D., J. Phys I France, 2 (1992) 1677-1690 ; Phys. Rev. B, 46 (1992) 7121-7126

[17] Papadia S., Piveteau B., Spanjaard D. et Desjonquères M.C., soumis à publication

[18] Wang S.C. et Ehrlich G., Surf. Sci., 239 (1990) 301-332

[19] Desjonquères M.C. et Spanjaard D., J. Phys. C, 15 (1982) 4007-4021

[20] Bassett D.W., Surf. Sci ., 53 (1975) 74-86; J. Phys. C, 9 (1976) 2491-2503

[21] Stoltze P. et Norskov J.K., Phys. Rev. B, 48 (1993) 5607-5611

[22] Liu C.L., Cohen J.M., Adams J.B. et Voter A.F., Surf. Sci., 253 (1991) 334-344

[23] Voir en particulier l'article de revue de Kellogg G.L., Appl. Surf. Sci., 87/88 (1995) 353-363

[24] Bassett D.W. et Webber P.R., Surf. Sci., 70 (1978) 520-531

[25] Feibelman P.J., Phys. Rev. Lett., 65 (1990) 729-732

[26] Hansen L., Stoltze P., Jacobsen K.W. et Norskov J.K., Phys. Rev. B, 44 (1991) 6523-6526

[27] Gravil P.A. et Holloway S., Surf. Sci., 310 (1994) 267-272

[28] Schwoebel R.L., J. Appl. Phys., 40 (1969) 614-618

Ehrlich G. et Hudda F.G., J. Chem. Phys., 44 (1966) 1039-1049

[29] Jardin J.P., Desjonquères M.C. et Spanjaard D., J. Phys. C, 18 (1985) 1767-1775

[30] Wang S.C. et Tsong T.T., Surf. Sci., 121 (1982) 85-97

[31] Bourdin J.P., Ganachaud J.P., Jardin J.P., Spanjaard D. et Desjonquères M.C., J. Phys. F, 18 (1988) 1801-1812

[32] Wang S.C. et Ehrlich G., Phys. Rev. Lett., 67 (1991) 2509-2512 